\title{
Assistência de enfermagem a um paciente com psicose não-orgânica e não específica: relato de experiência acadêmico
}

Nursing care for a patient with non-organic and non-specific psychosis: report of academic experience

Atención de enfermería a un paciente con psicosis no orgánica y no específica: relato de experiência académica

Luana Gomes de Lima Martins ORCID: https://orcid.org/0000-0002-8076-0397 Universidade do Estado do Pará, Brasil E-mail: luanagomesdelima7@gmail.com

Karollyne Quaresma Mourão ORCID: https://orcid.org/0000-0002-6242-1965 Universidade do Estado do Pará, Brasil E-mail: karollynemourao@hotmail.com

Ingrid Magali de Souza Pimentel ORCID: https://orcid.org/0000-0003-1820-5496 Universidade do Estado do Pará, Brasil E-mail: imbarleta@yahoo.com.br

Tatiana Menezes Noronha Panzetti ORCID: https://orcid.org/0000-0001-7560-4595 Universidade do Estado do Pará, Brasil E-mail: tnpanzetti@hotmail.com Ilma Pastana Ferreira

ORCID: https://orcid.org/0000-0002-9152-3872 Universidade do Estado do Pará, Brasil E-mail: pastanailma@gmail.com

Larissa Aline Costa Coelho ORCID: https://orcid.org/0000-0001-9764-3579 Universidade do Estado do Pará, Brasil E-mail: larissaalineccoelho@gmail.com

Margarete Feio Boulhosa

ORCID: https://orcid.org/0000-0003-1680-9368 Universidade do Estado do Pará, Brasil E-mail: margareteboulhosa@gmail.com

Dione Seabra de Carvalho ORCID: https://orcid.org/0000-0001-5342-6820 Faculdade Cosmopolita, Brasi E-mail: diseabra@bol.com.br Valquiria Rodrigues Gomes ORCID: https://orcid.org/0000-0003-2522-4441 Universidade do Estado do Pará, Brasil

E-mail: valquiria.rgomes@yahoo.com.br Ana Beatriz Capela Cordovil

ORCID: https://orcid.org/0000-0002-3080-6553 Universidade do Estado do Pará, Brasil E-mail: anabeatrizcapelac@gmail.com

Luanda da Silva e Silva

ORCID: https://orcid.org/0000-0001-7097-3068 Universidade do Estado do Pará, Brasil

E-mail: luandacat.crazy@gmail.com

Giovanna Mendonça Ribeiro

ORCID: https://orcid.org/0000-0002-8810-2655

Centro Universitário FIBRA, Brasil

E-mail: Gihmendonca10@gmail.com

Morianne Gomes da Mata

ORCID: https://orcid.org/0000-0001-6680-5010 Universidade do Estado do Pará, Brasil E-mail: moriannegomes@gmail.com 


\author{
Iasmim Ianne Sousa Tavares \\ ORCID: https://orcid.org/0000-0003-2350-874X \\ Universidade do Estado do Pará, Brasil \\ E-mail: iasmim.tavares14@gmail.com \\ Rainny Beatriz Saboia de Oliveira \\ ORCID: https://orcid.org/0000-0002-4086-2639 \\ Universidade do Estado do Pará, Brasil \\ E-mail: rainnysaboi7@gmail.com \\ Cristalia de Melo da Silva \\ ORCID: https://orcid.org/0000-0002-2449-387X \\ Universidade do Pará, Brasil \\ E-mail: cristaliademelo@gmail.com \\ Livia Caroline Machado da Silva \\ ORCID: https://orcid.org/0000-0002-7356-4005 \\ Universidade do Estado do Pará, Brasil \\ E-mail: liviamachados_@hotmail.com \\ Fabiane dos Santos Ferreira \\ ORCID: https://orcid.org/0000-0003-4484-8233 \\ Centro Universitário FIBRA, Brasil \\ E-mail: fabianedsferreira@gmail.com \\ Fabiana Morbach da Silva \\ ORCID: https://orcid.org/0000-0003-1583-0480 \\ Universidade do Estado do Pará, Brasil \\ E-mail: fabimorbach@gmail.com \\ João Victor Corrêa Pinto \\ ORCID: https://orcid.org/0000-0003-2513-4657 \\ Universidade Federal do Pará, Brasil \\ E-mail: JoaoVictorCorrea19990408Pinto@gmail.com
}

\title{
Resumo
}

Os transtornos psicóticos não-orgânicos não especificados, também conhecidos como F-29, são transtornos alucinatórios ou delirantes, assim como modificações de pensamento, passando a ser mais desorganizado, e de personalidade. $\mathrm{O}$ estudo tem como objetivo relatar a experiência de acadêmicos de enfermagem na Sistematização da Assistência de Enfermagem a um paciente com F-29 (psicose não-orgânica e não específica). Trata-se de um relato de experiência de natureza qualitativa, de aspecto descritivo, desenvolvido durante a prática do componente curricular de saúde mental II, realizado em um hospital de referência em atendimentos psiquiátricos no município de Belém-PA, no período de uma semana, com a utilização do arco de Marguerez. Após a coleta da primeira etapa do processo de enfermagem, foi possível ser traçados diagnósticos e intervenções a fim de proporcionar ao paciente melhor prognóstico e aprimorar a prática e assistência profissional aos acadêmicos. Com base no exposto, foi possível observar que a assistência de enfermagem dentro do processo patológico do paciente é de suma importância, principalmente no quesito de estímulo a sua melhora gradativa. Aliado a isso, foi possível verificar que os estudos referentes a essa patologia em questão ainda são poucos nas bases literárias, para um estudo mais aprofundado e com mais detalhamento de informações a respeito da mesma. Posto isso, foram pensadas sugestões para trabalhos futuros tais como: incorporação de aspectos fisiopatológicos, eficácia e impactos frente à atuação do enfermeiro e aspectos epidemiológicos da F-29.

Palavras-chave: Sistematização da assistência de enfermagem; Psicose; Assistência à saúde.

\begin{abstract}
Unspecified non-organic psychotic disorders, also known as F-29, are hallucinatory or delusional disorders, as well as changes in thinking, becoming more disorganized, and personality. The study aims to report the experience of nursing students in the Systematization of Nursing Care to a patient withF-29 (non-organic and non-specific psychosis). This is a report of an experience of a qualitative nature, with a descriptive aspect, developed during the practice of the curricular component of mental health II, carried out in a referral hospital for psychiatric care in the city of Belém-PA, within a week, using the Marguerez arch. After collecting the first stage of the nursing process, it was possible to draw up diagnoses and interventions in order to provide the patient with a better prognosis and improve the practice and professional assistance to academics.Based on the above, it was possible to observe that nursing care within the patient's pathological process is of paramount importance, especially in terms of encouraging its gradual improvement. Allied to this, it was possible to verify that the studies referring to this pathology in question are still few in the literary bases, for a more in-depth study and with more detail of information about it. That said, suggestions were made for future work such as:incorporation of pathophysiological aspects, effectiveness and impacts in face of the nurse's performance and epidemiological aspects of the F-29.
\end{abstract}

Keywords: Systematization of nursing care; Psychosis; Health care. 


\section{Resumen}

Los trastornos psicóticos no orgánicos no especificados, también conocidos como F-29, son trastornos alucinatorios o delirantes, así como cambios en el pensamiento, la desorganización y la personalidad. El estudio tiene como objetivo reportar la experiencia de estudiantes de enfermería en la Sistematización de la Atención de Enfermería a un paciente conF-29 (psicosis no orgánica y no específica). Se trata de un informe de una experiencia de carácter cualitativo, con aspecto descriptivo, desarrollada durante la práctica del componente curricular de salud mental II, realizada en un hospital de referencia para atención psiquiátrica en la ciudad de Belém-PA, dentro de una semana, utilizando el arco de Marguerez. Luego de recolectar la primera etapa del proceso de enfermería, fue posible elaborar diagnósticos e intervenciones con el fin de brindar al paciente un mejor pronóstico y mejorar la práctica y asistencia profesional a los académicos.Con base en lo anterior, se pudo observar que el cuidado de enfermería dentro del proceso patológico del paciente es de suma importancia, especialmente en términos de incentivar su mejora gradual. Aliado a esto, se pudo constatar que los estudios referentes a esta patología en cuestión son aún pocos en las bases literarias, para un estudio más profundo y con mayor detalle de información al respecto. Dicho esto, se hicieron sugerencias para trabajos futuros como:incorporación de aspectos fisiopatológicos, efectividad e impactos frente al desempeño del enfermero y aspectos epidemiológicos del F-29.
\end{abstract}

Palabras clave: Sistematización del cuidado de enfermeira; Psicoses; Cuidado de la salud.

\title{
1. Introdução
}

Os transtornos são definidos por variações do pensamento, afeto e comportamento que sempre perpetuaram ao decorrer da vida humana. Não obstante, outrora, tais transtornos eram tidos ora como presença demoníaca, ora como evidên cia dos deuses. Somado a isso, a psicose só foi considerada como doença, com a matéria para seu estudo e terapêutica - a psiquiatria -, realidade essa ocorrida no final do século XVIII com a Revolução Francesa (Zappaterra, 2012).

Psicose conceitua-se como um estado mental patológico descrito pela perda de contato com o individuo e a realidade, que passa a mostrar comportamentos anti-sociais. Relacionado a isso, os transtornos psicóticos não-orgânicos não especificados, também conhecido como F29, são transtornos alucinatórios ou delirantes, assim como modificações de pensamento, passando a ser mais desorganizado e, de personalidade, que não se incluem dentro da psicose funcional e psicose orgânica e, também não alegam os diagnósticos da esquizofrenia, mesmo com sintomas e características semelhantes. Identifica-se que esses tipos de transtornos pouco são encontrados em bases literárias que categorizem as características fidedignas da doença (Silva et al., 2018).

O diagnóstico dos transtornos mentais apresenta diversos fatores, entre eles os aspectos biológicos, ambientais e psicológicos. O diagnóstico estabelecido durante a primeira ocorrência da psicose tem importantes consequências tanto no tratamento quanto na prognose, e a partir disso é que são levantadas deliberação para a terapêutica (Araujo, Godoy \& Botti, 2017). Faz-se importante saber que, o diagnóstico e a intervenção inicial fornecem possibilidades para a implantação de medidas de prevenção de eventuais perdas e complicações relacionada à patologia, auxiliando na promoção da recuperação e redução da morbidade posterior. Programas específicos para terapêutica do episódio inicial psicótico estabelecem como suposições básicas a identificação precoce, a prevenção de recaídas e o tratamento intensivo. Estas ações possibilitam a diminuição do tempo da psicose não tratada e possibilitam a inclusão do tratamento intensivo do paciente e de seus familiares (Araujo, et al., 2017).

Dentro desse contexto, a assistência de enfermagem é de real importância, uma vez que, a mesma promove a projeção e manutenção do cuidado ao paciente. Em consonância a isso, a Sistematização da Assistência de Enfermagem (SAE) é um método científico que auxilia no trabalho dos enfermeiros possibilitando o planejamento e desempenho de tarefas a um paciente em específico, de acordo com suas limitações e necessidades, além de proporcionar ao paciente um cuidado mais humanizado e qualificado que ajude o mesmo no processo de transtorno mental (Mesquita \& Tavares, 2020; Xavier et al., 2020;). 
Com base no exposto supracitado, o trabalho objetiva relatar a experiência de acadêmicos de enfermagem na Sistematização da Assistência de Enfermagem a um paciente portador de um transtorno mental denominado de F29 (psicose não-orgânica e não específica).

\section{Metodologia}

Trata-se de um relato de experiência de natureza qualitativa, de aspecto descritivo, desenvolvido durante a prática do componente curricular de saúde mental II, realizado em um Hospital referência em atendimentos psiquiátricos no município de Belém-PA.

Segundo Pereira, Shitsuka, Parreira e Shitsuka (2018) as metodologias qualitativas são aquelas que representam a interpretação por parte do investigador/pesquisador, levando em consideração as suas contribuições sobre a situação em estudo. Nelas o processo de coleta de informações se da principalmente por meio de entrevistas com questionamentos abertos. Segundo os autores mencionados anteriormente, esse tipo de pesquisa apresenta algumas caracterizações, tais como: o pesquisador é o fundamental instrumento dentro desse processo, além de que a mesma acontece em meio natural com coleta de dados; as informações coletadas são preferivelmente descritivas; a inquietação do método é hegemônica em relação à do produto; o "conceito" que os indivíduos dão a vida e as coisas são alvos de atenção para o investigador e; as informações e a análise dos dados obtidos tende a acompanhar um processo indutivo.

Realizou-se a Sistematização da Assistência de Enfermagem (SAE) e o processo de enfermagem em um paciente que apresentava F29 (psicose não-orgânica e não específica), sendo pautado na teoria da problematização do arco de Maguerez que é constituído por cinco etapas: Observação da realidade; pontos chaves; teorização dos pontos chaves; construção de hipótese de solução e aplicação a realidade (Esperidão, Souza, Caixeta, Pinho \& Nunes, 2017).

A primeira etapa do arco de Marguerez iniciou no dia 4 de dezembro de 2019, durante o estágio da disciplina supracitada, onde foi possível os acadêmicos observarem numerosos pacientes com transtornos mentais, a partir disso foi escolhido um paciente para realização do estudo em questão que apresentava psicose não orgânica e não especificado, com necessidade de contínuos cuidados mais integral e humanizado, não só da equipe de enfermagem como também da equipe multiprofissional, em virtude de seu quadro clínico.

Embora a maior parte dos hospitais de grande porte detém da utilização da sistematização da assistência de enfermagem pelos profissionais enfermeiros, o que se observa atualmente é uma "mecanização" do cuidado ao paciente em suas diferentes demandas de cuidados, em especial, no ambiente desse estudo. Com isso pontuamos alguns pontos chaves: a ausência de um olhar mais humanizado por parte da equipe de enfermagem no uso da SAE, a falta de interação do paciente do estudo com outros pacientes e demandas psicológicas a serem melhoradas gradativamente (Sousa, Lima, Félix \& Souza 2020).

Após isso, foi plausível realizar o levantamento da teorização da temática abordada, que se refere à terceira etapa do arco, para melhor conhecer cientificamente a mesma através de buscas nas literaturas científicas. Aliado a isso, foi compreensível que uma das principais formas de melhora do quadro do paciente se dá a partir da realização de uma importante ferramenta de trabalho do enfermeiro, que é a SAE, se referindo não somente ao cuidado do paciente pelo enfermeiro, mas também com corroboração da equipe multiprofissional para efetivação e cuidado mais qualificado desse processo (Costa, 2012).

De acordo com os aspectos da problemática retratados nos pontos chaves, se apresentam de suma importância como hipóteses de solução a construção e execução da Sistematização da Assistência de Enfermagem a esse paciente, sendo seguidas as seguintes etapas: Histórico de enfermagem para coleta de informações que visam à elaboração dos diagnósticos de 
enfermagem; Diagnósticos de Enfermagem; planejamentos da assistência e resultados esperados; implementação da assistência e avaliação de enfermagem, além do apoio de todo corpo de profissionais da saúde.

Na quinta etapa que se refere à aplicação a realidade, os acadêmicos realizaram a construção da SAE, com base nas hipóteses de soluções levantadas, sendo feita a coleta de dados durante a primeira etapa da SAE que perpassaram por análise, embasando os diagnósticos de enfermagem realizados, resultados esperados e intervenções a serem realizadas.

Em consonância ao exposto, denota-se assim a experiência como uma fonte abundante de sentidos, objeto de análise do relato de experiência (RE) e perspectivas passíveis de análises. Com isso, há inúmeras alternativas narrato/descritivas provenientes dos encontros indivisíveis apresentados nos RE. Alguma coisa resta e é suscetível de ser reiterado e posto de outro modo, visto que, o RE é fruto de um acontecimento que percorreu pelo corpo de sue relator em um marcado período. O momento do relato marca sua articulação, está ligado às condições ideológicas, afetivas e as particularidades intersubjetivos com as suas significações socio-históricas. Dessa maneira, desarticula e não compatibiliza com um ponto de vista imutável e único (Daltro \& Faria, 2019).

\section{Resultados e Discussão}

A disciplina de Saúde Mental II, pertencente ao componente curricular dos acadêmicos em questão, proporcionou significativo conhecimento tanto teórico quanto prático, que viabilizou a prestação de cuidados e assistência nos serviços de alta complexidade em saúde.

Diante disso foi procedida a construção da SAE em uma paciente de 22 anos, pardo, do sexo masculino, solteiro, católico, que estudou até o $2^{\circ}$ grau completo, residente do município de Belém-PA. Internado na clínica psiquiátrica (PS) desde o dia 17/12/2020, acompanhado pela mãe, por demanda espontânea, com queixa principal de agitação motora, e com necessidade de contenção mecânica imediata. HDA: já apresentou 2 internações anteriores em relação ao seu estado patológico, com histórico de agressividade, solilóquios, pensamentos desorganizados, além de relato de tentativa de suicídio. Faz uso das referidos medicamentos: Risperidona - 1CP (2mg - 3x/ dia), pertencente à classe de medicamentos antipsicóticos atípicos; Prometazina - 1CP (25mg - 3x/dia), anti-histamínico; Levomepromazina - 3 CP(100mg - 1x/dia), antipsicótico; Ácido valpróico (500 mg - 3x/dia), anticonvulsivante e estabilizador do humor; Carbonato de lítio (300 mg - 3x/dia), estabilizador do humor; Omeprazol - 2CP (20mg - 1x/dia), é um medicamento protótipo da classe dos inibidores da bomba de protões, que diminuem a secreção gástrica alterando a atividade da H+/K+-ATPase; Diazepam 1 CP - (1x/dia), que é um hipnótico e ansiolítico. Ao exame mental realizado, o paciente encontrava-se deambulando no salão do hospital, aparentemente calmo, acessível ao diálogo, relatou evacuação e urina presente, além disso, o mesmo apresentava-se consciente, orientado, sem presença de alterações de sensopercepção, atenção preservada, memória prejudicada, inteligência prejudicada, pensamento com fulga de ideias e delirante, logorreico e bradilálico, hipomodulado, hipotímico, reservado e deambulando mais isoladamente.

Com base nos dados coletados (Histórico de enfermagem e evolução) foi possível realizar a sistematização da assistência de enfermagem a esse paciente, sendo elaborados os seguintes diagnósticos de enfermagem, resultados esperados e suas respectivas intervenções, apresentados no quadro1, a fim de melhorar a qualidade de vida do paciente, por meio das ações que foram planejadas e associadas aos cuidados da equipe multiprofissional. 
Quadro 1. Diagnósticos, resultados esperados e intervenções de enfermagem para o paciente em estudo.

\begin{tabular}{|c|c|c|}
\hline Diagnósticos & Resultados esperados & Intervenções de Enfermagem \\
\hline $\begin{array}{l}\text { Comunicação Verbal Prejudicada } \\
\text { relacionada ao efeito medicamentoso } \\
\text { para tratamento de sua psicose } \\
\text { evidenciado pela fala compassada e } \\
\text { de pouco entendimento }\end{array}$ & $\begin{array}{l}\text { - almeja-se a melhora no processo de } \\
\text { fala }\end{array}$ & $\begin{array}{l}\text { - manter ambiente calmo e quieto para } \\
\text { que o cliente possa concentrar-se no } \\
\text { esforço de comunicação e não tenha } \\
\text { que falar alto. } \\
\text { - ouvir com atenção. } \\
\text { - dar uma instrução a cada vez, } \\
\text { conforme apropriado. }\end{array}$ \\
\hline $\begin{array}{l}\text { Risco de Violência Direcionada a } \\
\text { outro relacionado à alteração na } \\
\text { função cognitiva e agressividade } \\
\text { secundária ao transtorno psicótico. }\end{array}$ & $\begin{array}{l}\text { - controle do comportamento de risco e } \\
\text { melhora da função cognitiva }\end{array}$ & $\begin{array}{l}\text { - estar em observação constante à } \\
\text { mudança do seu quadro clínico. } \\
\text { - estar atento ao conteúdo dos delírios e } \\
\text { do estado de humor. } \\
\text { - construir uma relação terapêutica de } \\
\text { confiança. }\end{array}$ \\
\hline $\begin{array}{l}\text { Interação Social Prejudicada } \\
\text { relacionada ao transtorno mental } \\
\text { (psicose) evidenciada por função } \\
\text { social prejudicada, ficando mais } \\
\text { isolado das outras pessoas. }\end{array}$ & $\begin{array}{l}\text { - almeja-se o desenvolvimento de } \\
\text { habilidades de interação social e } \\
\text { envolvimento social }\end{array}$ & $\begin{array}{l}\text { - estimular a convivência com os } \\
\text { demais pacientes. } \\
\text { - promover a resiliência. } \\
\text { - aconselhar e melhorar o sistema de } \\
\text { apoio, com o auxílio da equipe } \\
\text { multiprofissional. }\end{array}$ \\
\hline $\begin{array}{l}\text { Alteração nos Processos do } \\
\text { Pensamento relacionado aos delírios } \\
\text { secundários psicose evidenciado } \\
\text { pensamento desorganizado e com } \\
\text { fulga de ideias. }\end{array}$ & $\begin{array}{l}\text { - almeja-se a melhora na organização } \\
\text { do pensamento e poucas fulgas de } \\
\text { ideias. }\end{array}$ & $\begin{array}{l}\text { - observar para o conteúdo do } \\
\text { pensamento através da fala do paciente. } \\
\text { - observar o seu comportamento diante } \\
\text { das ideações delirantes. } \\
\text { - acionar médico e farmacêutico, caso } \\
\text { o paciente evolua com piora do quadro } \\
\text { delirante, para que ocorra a } \\
\text { medicalização do mesmo. }\end{array}$ \\
\hline $\begin{array}{l}\text { Memória Prejudicada relacionada ao } \\
\text { prejuízo que a psicose causa } \\
\text { evidenciada por incapacidade de } \\
\text { lembrar com veracidade fatos que } \\
\text { ocorreram anteriormente. }\end{array}$ & - melhora da cognição. & $\begin{array}{l}\text { - estimular a memória pela repetição do } \\
\text { último pensamento que o paciente } \\
\text { expressou. } \\
\text { - dar oportunidade para uso da memória } \\
\text { de eventos recentes. } \\
\text { - recordar experiências passadas com o } \\
\text { paciente, conforme apropriado. }\end{array}$ \\
\hline
\end{tabular}

Fonte: Adaptado de Herdman (2015), Nanda (2020) e Carpenito-Moyet (2008).

No Quadro 1 estão dispostos os diagnósticos, resultados esperados e intervenções de enfermagem, ou seja, parte de essencial importância no processo de construção da SAE ao paciente e, servindo como embasamento para prestação de cuidados ao mesmo.

Dentro do contexto de implantação da SAE foi possível observar como a mesma é uma ferramenta essencial no processo de trabalho dos enfermeiros, provendo a cientificidade dos traçados de cuidados a serem realizados, possibilitando a melhora do quadro e qualidade de vida do paciente em questão (Morais, Brasil, Tolfo, Costa \& Lohmann, 2020).

Aliado a isso, a etapa de avaliação de enfermagem não deixa de ser importante e fundamental, pois é a partir dela que se pode verificar a eficácia ou não das intervenções feitas e o que se pode modificar para uma intervenção que não apresentou eficácia na aplicação, além de possibilitar a reavaliação positiva, ou seja, retirar uma ação precedida que conseguiu ser atendida e resolvida (Mota, Silva \& Souza, 2016). 
A partir desta etapa pôde-se perceber uma pequena melhora no quadro do cliente, uma vez que, as fulgas de pensamento começaram a ocorrerem com menos intensidade, além dos fatos contados também apresentaram melhora em questão da veracidade. Somado a isso, a avaliação precisa ser contínua e frequentemente realizada, pois é a partir dela que se terá um feedback da situação geral de um paciente.

Para efetividade da prestação de cuidados a um paciente é imprescindível à atuação de toda equipe multiprofissional de saúde, seja ela de atenção hospital ou básica, pois é através da junção de cuidados da equipe que se pode realmente observar a efetividade do serviço prestado e ofertado (Santos, Pestana, Guerrero, Meirelles \& Erdmann, 2013).

Oliveira, Almeida, Moreira e Torres (2019) também descrevem que a SAE é uma ferramenta teórica que quando aplicada na prática tem maior efeito e resultado, como visto em sua construção e aplicação ao paciente do estudo. Por meio dela os acadêmicos observaram a sua eficácia e como a mesma se constrói frente a um paciente com transtorno mental.

A partir da prática ocorrida, verificou-se que a vivência proporcionada pelo componente curricular saúde Mental II foi de significativa relevância para o aprimoramento e conhecimento dos acadêmicos sobre o assistir da equipe e unidade de saúde, além de promover os cuidados apreendidos em sala de aula. Diante disso, o relato de experiência se propõe como uma metodologia que busca compartilhar os conhecimentos e aprendizados adquiridos, principalmente, na vivência prática de uma disciplina, o que evidência a sua e importância e influenciam diante das práticas curriculares de formação (Morais et al., 2020).

\section{Conclusão}

Com base no exposto, foi possível observar que a assistência de enfermagem dentro do processo patológico do paciente é de suma importância, principalmente no quesito de estímulo a sua melhora gradativa. Aliado a isso, observamos e constatamos que os estudos referentes a essa patologia, ainda são poucos nas bases cientificas, para um estudo mais aprofundado e com mais detalhamento de informações a respeito da mesma. Sendo necessário investimento em estudos científicos relacionado ao assunto estudado, a embasar a assistência de enfermagem ao acometido de transtorno mental F29 (psicose não-orgânica e não específica).

Como já mencionado anteriormente, o levantamento dos estudos nas bases bibliográficas referentes à patologia em questão se mostrou escassos. Em função disso, recomenda-se para trabalhos futuros a incorporação de aspectos fisiopatológicos mais aprofundados e direcionados, além de estudos que verifiquem a eficácia e impactos na atuação do profissional enfermeiro frente à sistematização da assistência de enfermagem, relacionados a F-29. E por fïm, são sugeridos estudos que abarquem mais o aspecto epidemiológico, posto que, nesse quesito existem poucas contribuições cientificas a cerca da mesma.

\section{Referências}

Araújo, L. M. C., Godoy, E. F. M., \& Botti, N. C. L. (2017). Situações presentes na crise de pacientes psicóticos. Arquivos Brasileiros de Psicologia, 69(2), 138-152. http://pepsic.bvsalud.org/scielo.php?script=sci_arttext\&pid=S180952672017000200010\&lng=en\&nrm=iso.

Carpenito-Moyet, L. J. (2008). Manual de Diagnósticos de Enfermagem: Artmed.

Costa, A. M., (2012). Importância da implementação da assistência de enfermagem (sae): uma abordagem bibliográfica: 2000 - 2012 (Artigo apresentado para o curso de pós graduação em Gestão Pública Em Saúde [EAD] da Universidade Federal de Santa Maria, RS). UFSM, 1-15. https://repositorio.ufsm.br/bitstream/handle/1/1459/Costa_Adonai_Mejia.pdf?sequence=1.

Daltro, M. R., \& Faria, A. A. (2019). Relato de experiência: Uma narrativa científica na pós-modernidade. Estudos e Pesquisas em Psicologia, $19(1), 223-237$. http://pepsic.bvsalud.org/scielo.php?script=sci_arttext\&pid=S1808-42812019000100013\&lng=pt\&tlng=pt.

Esperidão, E., Souza, A., Caixeta, C. C., Pinho, E. S., \& Nunes, F. C. (2017). Arco de Maguerez: estratégia de metodologia ativa para coleta de dados. (2017). In: Congresso Inbero-Americano Em Investigação Qualitativa (6 ${ }^{\circ}$ CIAIQ), Salamanca. Atas...Salamanca: Investigação Qualitativa, v. 2. 825-834. https://proceedings.ciaiq.org/index.php/ciaiq2017/article/view/1279/1239.

Herdman, T. H. (2015). Diagnósticos de enfermagem da NANDA: definições e classificação 2015-2017: Artmed. 
Research, Society and Development, v. 10, n. 2, e8810212274, 2021 (CC BY 4.0) | ISSN 2525-3409 | DOI: http://dx.doi.org/10.33448/rsd-v10i2.12274

Morais, G. J., Brasil, T. C., Tolfo, G. R., da Costa, A. E. K., \& Lohmann, P. M. (2020). A Sistematização da Assistência de Enfermagem (SAE) a um paciente com obesidade e hipertensão: relato de experiência. Research, Society and Development, 9(9), p. 1-10, e962997940. https://doi.org/10.33448/rsd-v9i9.7940.

Mota, A. S., Silva, A. L. A., \& Souza, Â. C. (2016). Educação permanente: Práticas e processos da enfermagem em saúde mental. Revista Portuguesa de Enfermagem de Saúde Mental, (spe4), 9-16. https://dx.doi.org/10.19131/rpesm.0135.

Mesquita, L. M. F. de \& Tavares, C. M. de M. (2020). Sistematização da Assistência de Enfermagem em Saúde Mental na Atenção Básica: Revisão Integrativa da Literatura. Revista Enfermagem Atual In Derme, 91(29), 124-130. https://doi.org/10.31011/reaid-2020-v.91-n.29-art.82p.

Nanda, International. (2020). Welcome to NANDA International. https://nanda.org/.

Pereira, A. S., Shitsuka, D. M., Parreira, F. J., \& Shitsuka, R. Metodologia da pesquisa científica [recurso eletrônico]. (2018). - (1. ed.): UFSM, NTE. 1 ebook. https://repositorio.ufsm.br/bitstream/handle/1/15824/Lic_Computacao_Metodologia-Pesquisa-Cientifica.pdf?sequence=1

Santos, J. L. G., Pestana, A. L., Guerrero, P., Meirelles, B. S. H., \& Erdmann, A. L. (2013). Práticas de enfermeiros na gerência do cuidado em enfermagem e saúde: revisão integrativa. Revista Brasileira de Enfermagem, 66(2), 257-263. https://dx.doi.org/10.1590/S0034-71672013000200016.

Silva, V. C. L., Portela, D. A., Silva, M. G. D. G., Pinheiro, C. P., Rodrigues, A. C. S., \& Carvalho, C. N. (2018). Análise clínica e farmacológica em um paciente portador de psicose não-orgânica não especificada (F29). In: Conexão Fametro. Fortaleza/CE. https://www.doity.com.br/anais/conexaofametro2018/trabalho/71097.

Sousa B. V. N., Lima C. F. M., Félix N. D. C., \& Souza F. O. (2020). Benefícios e limitações da sistematização da assistência de enfermagem na gestão em saúde. J. nurs. Health. 10(2): e20102001. https://periodicos.ufpel.edu.br/ojs2/index.php/enfermagem/article/view/15083/11184.

Zappaterra, F. A. (2012). Assistência de enfermagem às pessoas com transtorno psicótico (Trabalho de Conclusão de Curso, Faculdade de Medicina de Marília) - Secretaria de Estado da Saúde-Fundap: [s.n.]. https://docs.bvsalud.org/biblioref/ses-sp/2012/ses-31176/ses-31176-2939.pdf.

Xavier, S. C. da M., Soeiro, R. de L., Mesquita, L. M., Lobo, B. M. I. da S., Valente, G. S. C., \& Cortez, E. A. (2020). Educ ação permanente e sistematização da assistência de enfermagem para atenção psicossocial. Research, Society and Development, 9(4), e56942839. https://doi.org/10.33448/rsd-v9i4.2839.

Oliveira, M. R., Almeida, P. C., Moreira, T. M. M., \& Torres, R. A. M. (2019). Sistematização da assistência de enfermagem: percepção e conhecimento da enfermagem Brasileira. Revista Brasileira de Enfermagem, 72(6), 1547-1553.https://doi.org/10.1590/0034-7167-2018-0606. 